\title{
Keller-Osserman a priori estimates and removability result for the anisotropic porous medium equation with absorption term
}

\author{
MARIA A. SHAN \\ (Presented by A. E. Shishkov)
}

\begin{abstract}
In this article we obtained the removability result for quasilinear equations model of which is

$$
u_{t}-\sum_{i=1}^{n}\left(u^{m_{i}-1} u_{x_{i}}\right)_{x_{i}}+f(u)=0, u \geq 0 .
$$

and prove a priori estimates of Keller-Osserman type.

2010 MSC. 35B40, 35B45.

Key words and phrases. Anisotropic porous medium equation, KellerOsserman a priori estimates, removability of isolated singularity.
\end{abstract}

\section{Introduction and main results}

In this paper we study solutions to quasilinear parabolic equation in the divergent form

$$
u_{t}-\operatorname{div} A(x, t, u, \nabla u)+a_{0}(u)=0,(x, t) \in \Omega_{T}=\Omega \times(0, T),
$$

satisfying a initial condition

$$
u(x, 0)=0, \quad x \in \Omega \backslash\{0\},
$$

where $\Omega$ is a bounded domain in $R^{n}, n \geq 2,0<T<\infty$.

We suppose that the functions $A=\left(a_{1}, \ldots, a_{n}\right)$ and $a_{0}$ satisfy the Caratheodory conditions and the following structure conditions hold

$$
A(x, t, u, \xi) \xi \geq \nu_{1} \sum_{i=1}^{n}|u|^{m_{i}-1}\left|\xi_{i}\right|^{2}
$$

Received 02.03.2018 


$$
\begin{gathered}
\left|a_{i}(x, t, u, \xi)\right| \leq \nu_{2} u^{\left(m_{i}-1\right) \frac{1}{2}}\left(\sum_{j=1}^{n}|u|^{m_{j}-1}\left|\xi_{j}\right|^{2}\right)^{\frac{1}{2}}, i=\overline{1, n}, \\
a_{0}(u) \geq \nu_{1} f(u),
\end{gathered}
$$

with positive constants $\nu_{1}, \nu_{2}$ and continuous, positive function $f(u)$ and

$$
\min _{1 \leq i \leq n} m_{i}>1-\frac{2}{n}, \max _{1 \leq i \leq n} m_{i} \leq m+\frac{2}{n},
$$

where $m=\frac{1}{n} \sum_{i=1}^{n} m_{i}$. Without loss of generality we will assume later that $m_{1} \leq m_{2} \leq \ldots \leq m_{n}$.

Many authors studied problems of singularities of solutions of second order quasilinear elliptic and parabolic equations. Review of these results can be found in the monograph of Veron [19]. Brezis and Veron [2] proved that for $q \geq \frac{n}{n-2}$ the isolated singularities of solutions to the elliptic equation

$$
-\triangle u+u^{q}=0,
$$

are removable. In [3] Brézis and Friedman proved that for $q \geq \frac{n+2}{n}$ the isolated singularities of solutions for the following parabolic equation

$$
\frac{\partial u}{\partial t}-\triangle u+|u|^{q-1} u=0, \quad(x, t) \in \Omega_{T} \backslash\{(0,0)\}
$$

are removable. The removability of isolated singularity for solutions of the nonanisotropic porous medium equation $\left(m=m_{1}=\ldots=m_{n},\right)$

$$
u_{t}-\triangle\left(|u|^{m-1} u\right)+|u|^{q-1} u=0,
$$

has been proved under the assumption $q \geq m+\frac{2}{n}$ by Kamin and Peletier [5].

Development of the qualitative theory of second order quasilinear elliptic and parabolic equations with nonstandart growth conditions has been observed in recent decades. Some results of $[4,6,7,9,10,12,15-18]$ we mention here. One of the example of such equations is

$$
\frac{\partial u}{\partial t}-\sum_{i=1}^{n} \frac{\partial}{\partial x_{i}}\left(|u|^{\left(m_{i}-1\right)\left(p_{i}-2\right)}\left|\frac{\partial u}{\partial x_{i}}\right|^{p_{i}-2} \frac{\partial u}{\partial x_{i}}\right)=0, p_{i} \geq 2, m_{i} \geq 1, i=\overline{1, n} .
$$

The removability result of isolated singularity and a priori estimates of Keller-Osserman type for this equation was obtained in [9, 12]. 
We now define a weak solution of the problem (1.1), (1.2) with singularity at the point $(0,0)$. We will write $V_{2, m}\left(\Omega_{T}\right)$ for the class of functions $\varphi \in C_{l o c}\left(0, T, L_{l o c}^{1+m^{-}}(\Omega)\right)$ with $\sum_{i=1}^{n} \iint_{\Omega_{T}}|\varphi|^{m_{i}+m^{-}-2}\left|\varphi_{x_{i}}\right|^{2} d x d t<\infty$, where $m^{-}=\min \left(m_{n}, 1\right)$. By a weak solution of the problem (1.1), (1.2) we mean a function $u(x, t) \geq 0$ satisfying the inclusion $u \psi \in$ $V_{2, m}\left(\Omega_{T}\right) \cap L_{l o c}^{2}\left(0, T ; W_{l o c}^{1,2}(\Omega)\right)$ and the integral identity

$$
\begin{gathered}
\int_{\Omega} u(x, \tau) \psi \varphi d x \\
+\int_{0}^{\tau} \int_{\Omega}\left\{-u(\psi \varphi)_{t}+A(x, t, u, \nabla u) \nabla(\psi \varphi)+a_{0}(u) \psi \varphi\right\} d x d t=0
\end{gathered}
$$

holds for any testing function $\varphi \in W_{l o c}^{1,2}\left(0, T ; L_{l o c}^{2}(\Omega)\right) \cap L_{l o c}^{2}\left(0, T ; \stackrel{o}{W}_{l o c}^{1,2}(\Omega)\right.$, any $\psi \in C^{1}\left(\bar{\Omega}_{T}\right)$ vanishing in the neighborhood of $\{(0,0)\}$ and for all $\tau \in(0, T)$.

The result of this paper is the removability of isolated singularities for solutions of the anisotropic porous medium equation with absorption term. The proof of this result is based on a priori estimates of KellerOsserman type of the solution to the equation (1.1). The main difficulty lies in the fact that part of $m_{i}<1$ (singular case), and another part of $m_{i}>1$ (degenerate case).

Theorem 1.1. Let the conditions (1.3), (1.4) be fulfilled and $u$ be a nonnegative weak solution to the problem (1.1), (1.2). Assume also that $f(u)=u^{q}$ and

$$
q \geq m+\frac{2}{n}
$$

then the singularity at the point $\{(0,0)\}$ is removable.

Let $\left(x^{(0)}, t^{(0)}\right) \in \Omega_{T}$, for any $\tau, \theta_{1}, \theta_{2}, \ldots, \theta_{n}>0, \theta=\left(\theta_{1}, \ldots, \theta_{n}\right)$ we define $Q_{\theta, \tau}\left(x^{(0)}, t^{(0)}\right):=\left\{(x, t):\left|t-t^{(0)}\right|<\tau,\left|x_{i}-x_{i}^{(0)}\right|<\theta_{i}, i=\overline{1, n}\right\}$ and set $M(\theta, \tau):=\sup _{Q_{\theta, \tau}\left(x^{\left.(0), t^{(0)}\right)}\right.} u, \quad F(\theta, \tau):=\sup _{Q_{\theta, \tau}\left(x^{(0)}, t^{(0)}\right)} F(u), F(u)=\int_{0}^{u} s^{m^{-}-1}$ $f(s) d s, m^{+}=\max \left(m_{n}, 1\right)$.

Theorem 1.2. Let the conditions (1.3), (1.4) be fulfilled and $u$ be a nonnegative weak solution to equation (1.1), assume also that $f \in C^{1}\left(R_{+}^{1}\right)$ and $f^{\prime}(u) \geq 0$. Let $\left(x^{(0)}, t^{(0)}\right) \in \Omega_{T}$, fix $\sigma \in(0,1)$, let $Q_{8 \theta, 8 \tau}\left(x^{(0)}, t^{(0)}\right) \subset$ 
$\Omega_{T}$. Set $\rho=\left\{\begin{array}{l}\theta_{n}, \text { if } m_{n}>1, \\ \tau^{\frac{1}{2}}, \text { if } m_{n}<1,\end{array}\right.$, then there exist positive number $c_{1}, c_{2}$ depending only on $n, \nu_{1}, \nu_{2}, m_{1}, \ldots, m_{n}$ such that either

$$
u\left(x^{(0)}, t^{(0)}\right) \leq\left(\frac{\theta_{n}^{2}}{\tau}\right)^{\frac{1}{m_{n}-1}}+\sum_{i=1}^{n-1}\left(\frac{\rho}{\theta_{i}}\right)^{\frac{2}{m^{+}-m_{i}}},
$$

or

$$
\begin{aligned}
& (M(\sigma \theta, \sigma \tau))^{1-m^{-}+\frac{n\left(m-m^{-}\right)}{2}} F(M(\sigma \theta, \sigma \tau)) \\
\leq & c_{1}(1-\sigma)^{-\gamma} \rho^{-2}(M(\theta, \tau))^{m^{+}+1+\frac{n\left(m-m^{-}\right)}{2}}
\end{aligned}
$$

holds true.

We also have, in particular, if

$$
F(\varepsilon u) \leq \varepsilon^{m^{+}+m^{-}+\beta} F(u), \beta>0,
$$

then

$$
F(M(\theta, \tau)) \leq c_{2}(1-\sigma)^{-\gamma} M^{m^{+}+m^{-}}(\theta, \tau) \rho^{-2},
$$

An example of the function $f$, which satisfies the conditions (1.9) is $f(u)=u^{q}, q \geq m+\frac{2}{n}$. Assuming for simplicity that $\operatorname{dist}\left(x^{(0)}, \partial \Omega\right)=\left|x^{(0)}\right|$, and choosing $\tau, \theta_{i}$, from the conditions

- $m_{n}>1:\left(\frac{\theta_{n}^{2}}{\tau}\right)^{\frac{1}{m_{n}-1}}=\theta_{n}^{-\frac{2}{q-m_{n}}}$, i.e. $\tau=\theta_{n}^{\frac{2(q-1)}{q-m_{n}}}$, $\left(\frac{\theta_{n}}{\theta_{i}}\right)^{\frac{2}{m_{n}-m_{i}}}=\theta_{n}^{-\frac{2}{q-m_{n}}}$, i.e. $\theta_{i}=\theta_{n}^{\frac{q-m_{i}}{q-m_{n}}}$

- $m_{n}<1:\left(\frac{\theta_{n}^{2}}{\tau}\right)^{\frac{1}{m_{n}-1}}=\tau^{-\frac{1}{q-m_{n}}}$, i.e. $\tau=\theta_{n}^{\frac{2(q-1)}{q-m_{n}}}$ $\left(\frac{\tau^{\frac{1}{2}}}{\theta_{i}}\right)^{\frac{2}{1-m_{i}}}=\tau^{-\frac{1}{q-1}}$, i.e. $\theta_{i}=\tau^{\frac{q-m_{i}}{2(q-1)}}$,

from $(1.7),(1.10)$ we obtain an estimate

$$
u\left(x^{(0)}, t^{(0)}\right) \leq c\left(\sum_{i=1}^{n}\left|x_{i}^{(0)}\right|^{\frac{2}{q-m_{i}}}+\left(t^{(0)}\right)^{\frac{1}{q-1}}\right)^{-1} .
$$

\section{Keller-Osserman a priori estimates}

\subsection{Auxiliary propositions}

Let $E(2 \rho)=\left\{(x, t) \in \Omega_{T}: u(x, t)>M(2 \rho)\right\}, u^{(\rho)}(x, t)=\min \left(M\left(\frac{\rho}{2}\right)-\right.$ $M(2 \rho), u(x, t)-M(2 \rho))$. 
Lemma 2.1. [11] Under the assumptions of Theorem 1.1 following inequality holds

$$
\begin{gathered}
\iint_{E(2 \rho)} u^{(\rho)} u^{q} \psi_{r}^{l} d x d t \leq \gamma\left(M\left(\frac{\rho}{2}\right)-M(2 \rho)\right) \\
\times\left\{F_{3}(r, \lambda)+\left(F_{1}(r, \lambda)+F_{2}(r, \lambda)\right)^{\frac{1}{2}} F_{4}^{\frac{1}{2}}(r, \lambda)\right\},
\end{gathered}
$$

where

$$
\begin{aligned}
& F_{1}(r, \lambda)=\left\{\begin{array}{r}
R^{\lambda}(r), \lambda>0, \\
\ln \frac{q-2}{q-1} \frac{1}{r}, \lambda=0, \quad q>2, \\
\ln \ln \frac{1}{r}, \lambda=0, \quad q=2, \\
\ln \frac{-2-q}{q-1} \frac{1}{r}, \lambda=0, \quad q<2
\end{array}\right. \\
& F_{2}(r, \lambda)=\left\{\begin{array}{r}
R^{\lambda}(r), \lambda>0, \\
\ln \frac{q-2 m_{1}}{q-m_{1}} \frac{1}{r}, \lambda=0, \quad q>2 m_{1}, \\
\ln \ln \frac{1}{r}, \lambda=0, \quad q=2 m_{1}, \\
\ln ^{-\frac{2 m_{1}-q}{1-m_{1}}} \frac{1}{r}, \lambda=0, \quad q<2 m_{1} .
\end{array}\right. \\
& F_{3}(r, \lambda)=\left\{\begin{array}{c}
R^{\lambda}(r), \lambda>0, \quad R_{4}^{\lambda}(r, \lambda)=\left\{\begin{array}{c}
R^{\lambda}(r), \lambda>0, \\
\ln ^{-1} \frac{1}{R(r)}, \lambda=0,
\end{array}\right.
\end{array}\right.
\end{aligned}
$$

where $\lambda=n-\frac{2}{q-m}, 0<r<R_{0}$.

Lemma 2.2. [1] Let $\Omega \subset R^{n}, n \geq 2$ be a bounded domain, $v \in \stackrel{o}{W}^{1,1}(\Omega)$ and

$$
\sum_{i=1}^{n} \int_{\Omega}|v|^{\alpha_{i}}\left|v_{x_{i}}\right|^{p_{i}} d x<\infty, \alpha_{i} \geq 0, p_{i}>1 .
$$

If $1<p<n$, then $v \in L^{q}(\Omega), q=\frac{n p}{n-p}\left(1+\frac{1}{n} \sum_{i=1}^{n} \frac{\alpha_{i}}{p_{i}}\right), \frac{1}{p}=\frac{1}{n} \sum_{i=1}^{n} \frac{1}{p_{i}}$ and the following inequality holds

$$
\|v\|_{L^{q}(\Omega)} \leq \gamma \prod_{i=1}^{n}\left(\int_{\Omega}|v|^{\alpha_{i}}\left|v_{x_{i}}\right|^{p_{i}} d x\right)^{\frac{1}{n p_{i}\left(1+\frac{1}{n} \sum_{i=1}^{n} \frac{\alpha_{i}}{p_{i}}\right)}},
$$

where the positive constant $\gamma$ depends only on $n, p_{i}, \alpha_{i}, i=\overline{1, n}$. 
Lemma 2.3. [8, chap. 2] Let $\left\{y_{j}\right\}_{j \in N}$ be a sequence of nonnegative numbers such that for any $j=0,1,2, \ldots$ the inequality

$$
y_{j+1} \leq C b^{j} y_{j}^{1+\varepsilon}
$$

holds with positive $\varepsilon, C>0, b>1$. Then the following estimate is true

$$
y_{j} \leq C^{\frac{(1+\varepsilon)^{j}-1}{\varepsilon}} b^{\frac{(1+\varepsilon)^{j}-1}{\varepsilon^{2}}-\frac{j}{\varepsilon}} y_{0}^{(1+\varepsilon)^{j}} .
$$

Particulary, if $y_{0} \leq C^{-\frac{1}{\varepsilon}} b^{-\frac{1}{\varepsilon^{2}}}$, then $\lim _{j \rightarrow \infty} y_{j}=0$.

\subsection{Integral estimates of solutions}

Consider a cylinder $Q_{\theta, \tau}\left(x^{(0)}, t^{(0)}\right)$ and let $(\bar{x}, \bar{t})$ be an arbitrary point in $Q_{\sigma \theta, \sigma \tau}\left(x^{(0)}, t^{(0)}\right)$. If $u\left(x^{(0)}, t^{(0)}\right) \geq\left(\frac{\theta_{n}^{2}}{\tau}\right)^{\frac{1}{m_{n}-1}}+\sum_{i=1}^{n-1}\left(\frac{\rho}{\theta_{i}}\right)^{\frac{2}{m^{+}-m_{i}}}$ then $M(\theta, \tau)=\max (M(\theta, \tau), \delta(\theta, \tau)) \geq\left(\tau^{-1} \theta_{n}\right)^{\frac{1}{m_{n}-1}}+\sum_{i=1}^{n}\left(\theta_{i}^{-1} \rho\right)^{\frac{2}{m^{+}-m_{i}}}$, and hence $Q_{\eta, s}(\bar{x}, \bar{t}) \subset Q_{\theta, \tau}\left(x^{(0)}, t^{(0)}\right)$, where $s=(1-\sigma) M^{1-m^{+}}(\theta, \tau) \rho^{2}, \eta_{i}=(1-$ $\sigma) M^{\frac{m_{i}-m^{+}}{2}}(\theta, \tau) \rho, i=\overline{1, n}$. For fixed $k>0$ and $l, j=0,1,2 \ldots$ set $\alpha_{l}=$ $\frac{1}{4}\left(1+2^{-1}+\cdots+2^{-l}\right)$, set $k_{j}=k\left(1-2^{-j}\right), \eta_{i, j, l}=\left(\alpha_{l}+\frac{1}{4} 2^{-j-l-1}\right) \eta_{i}, i=$ $\overline{1, n}, \eta_{j, l}=\left(\eta_{1, j, l}, \ldots, \eta_{n, j, l}\right), s_{j, l}=\left(\alpha_{l}+\frac{1}{4} 2^{-j-l-1}\right) s, Q_{j, l}=Q_{\eta_{j, l}, s_{j, l}}(\bar{x}, \bar{t})$, $A_{k_{j}, j, l}=\left\{x \in Q_{j, l}(\bar{x}, \bar{t}): F(u)>k_{j}\right\}$. Let $\xi_{j} \in C_{0}^{\infty}\left(Q_{j, l}(\bar{x}, \bar{t})\right), 0 \leq \xi_{j} \leq$ $1, \xi_{j}=1$ in $Q_{j+1, l}(\bar{x}, \bar{t}),\left|\frac{\partial \xi_{j}}{\partial t}\right| \leq \gamma 2^{j+l} s^{-1},\left|\frac{\partial \xi_{j}}{\partial x_{i}}\right| \leq \gamma 2^{j+l} \eta_{i}^{-1}, i=\overline{1, n}$.

In what follows $\gamma$ stands for a constant depending only $n, \nu_{1}, \nu_{2}$, $m_{1}, \ldots, m_{n}$ which may vary from line to line.

Lemma 2.4. Let $u$ be a nonnegative weak solution to equation (1.1) and let conditions (1.3), (1.4) hold. Then for any $j \geq 0$ the following inequality holds true

$$
\begin{gathered}
l_{j}^{1-m^{-}} \int_{A_{k_{j}, j, l}(t)}\left(F(u)-k_{j}\right)_{+}^{2} \xi_{j}^{2} d x+\sum_{i=1}^{n} l_{j}^{m_{i}-m^{-}} \iiint_{A_{k_{j}, j, l}}\left|\nabla\left(\left(F(u)-k_{j}\right)_{+}\right)\right|^{2} \xi_{j}^{2} d x d t \\
+\iint_{A_{k_{j}, j, l}}\left(F(u)-k_{j}\right)_{+} f^{2}(u) \xi_{j}^{2} d x d t \leq \gamma M^{m^{+}-m^{-}}(\theta, \tau) \rho^{-2} \iint_{A_{k_{j}, j, l}}\left(F(u)-k_{j}\right)_{+}^{2} d x d t
\end{gathered}
$$

where $l_{j}=F^{-1}\left(k_{j}\right), j=0,1,2, \ldots$ 
Proof. Testing identity $(1.5)$ by $\varphi=\left(F(u)-k_{j}\right)_{+} f(u) \xi_{j}^{2}$, using conditions (1.3) we obtain

$$
\begin{gathered}
\iint u_{t} f(u)\left(F(u)-k_{j}\right)_{+} \xi_{j}^{2} d x d t \\
+\sum_{i=1}^{n} \iint u_{A_{k_{j}, j, l}} u^{m_{i}+m^{-}-2}\left|u_{x_{i}}\right|^{2} f^{2}(u) \xi_{j}^{2} d x d t+\iint\left(F(u)-k_{j}\right)_{+} f^{2}(u) \xi_{j}^{2} d x d t \\
\leq \gamma \sum_{i=1}^{n} \iint_{A_{k_{j}, j, l}, l} u^{\frac{m_{i}-1}{2}}\left(\sum_{l=1}^{n} u^{m_{l}-1}\left|u_{x_{l}}\right|^{2}\right)^{\frac{1}{2}}\left(F(u)-k_{j}\right)_{+} f(u) \xi_{j}\left|\frac{\partial \xi_{j}}{\partial x_{i}}\right| d x d t .
\end{gathered}
$$

From this, using the Young inequality and the evident inequality $l_{j}<u(x, t)<M(\theta, \tau)$ on $A_{k_{j}, j, l}$ we arrive at the required (2.4).

\subsection{Proof of Theorem 1.2}

By Lemma 2.2 and the Hölder inequality we obtain

$$
\begin{aligned}
& Y_{j+1, l}=\iint_{A_{k_{j+1}, j+1, l}}\left(F(u)-k_{j+1}\right)_{+}^{2} d x d t \\
& \leq\left|A_{k_{j+1}, j+1, l}\right|^{\frac{2}{n+2}}\left(\iiint_{A_{k_{j+1}, j+1, l}}\left(\left(F(u)-k_{j+1}\right)_{+} \xi_{j}\right)^{\frac{2(n+2)}{n}} d x d t\right)^{\frac{n}{n+2}} \\
& \leq\left|A_{k_{j+1}, j+1, l}\right|^{\frac{2}{n+2}} \operatorname{ess}_{0<t<T}\left(\int_{A_{k_{j+1}, j+1, l}(t)}\left(F(u)-k_{j+1}\right)_{+}^{2} \xi_{j}^{2} d x\right)^{\frac{2}{n+2}} \\
& \times\left(\int_{0}^{T} \prod_{i=1}^{n}\left(\int_{A_{k_{j+1}, j+1, l}(t)}\left|\left(\left(F(u)-k_{j+1}\right)+\xi_{j}\right)_{x_{i}}\right|^{2} d x\right)^{\frac{1}{n}} d t\right)^{\frac{n}{n+2}} \\
& \leq\left|A_{k_{j+1}, j+1, l}\right|^{\frac{2}{n+2}} \operatorname{ess}_{0<t<T}\left(\int_{A_{k_{j+1}, j+1, l}(t)}\left(F(u)-k_{j+1}\right)_{+}^{2} \xi_{j}^{2} d x\right)^{\frac{2}{n+2}}
\end{aligned}
$$




$$
\begin{aligned}
& \times\left(\int _ { 0 } ^ { T } \prod _ { i = 1 } ^ { n } \left(\int_{A_{k_{j+1}, j+1, l}(t)}\left|\left(\left(F(u)-k_{j+1}\right)_{+}\right)_{x_{i}}\right|^{2} \xi_{j}^{2} d x\right.\right. \\
& \left.\left.+\int_{A_{k_{j+1}, j+1, l}(t)}\left(F(u)-k_{j+1}\right)_{+}^{2}\left|\frac{\partial \xi_{j}}{\partial x_{i}}\right|^{2} d x\right)^{\frac{1}{n}} d t\right)^{\frac{n}{n+2}} .
\end{aligned}
$$

Denote $Q_{l}=Q_{\alpha_{l} \eta, \alpha_{l} s}, M_{l}=\sup _{Q_{l}} u$, using (2.4), it follows from Lemma 2.3 that $y_{j, l} \rightarrow 0$ as $j \rightarrow \infty$, provided $k$ is chosen to satisfy

$$
k^{2}=\gamma 2^{l \gamma} l_{j}^{m^{-}-1+\frac{n\left(m^{-}-m\right)}{2}} M_{l+1}^{\frac{(n+2)\left(m^{+}-m^{-}\right)}{2}}(\theta, \tau) \rho^{-n-2} \iint_{Q_{l+1}} F^{2}(u) d x d t .
$$

From this we obtain

$$
\begin{gathered}
M_{l}^{1-m^{-}+\frac{n\left(m-m^{-}\right)}{2}} F^{2}\left(M_{l}\right) \\
\leq \gamma(1-\sigma)^{-\gamma} 2^{l \gamma} M_{l+1}^{\frac{(n+2)\left(m^{+}-m^{-}\right)}{2}} \rho^{-n-2} \iint_{Q_{l+1}} F^{2}(u) d x d t .
\end{gathered}
$$

Denoting $M_{l}^{\frac{1-m^{-}}{2}+\frac{n\left(m-m^{-}\right)}{4}} F\left(M_{l}\right)=M_{l}^{\frac{a}{2}} F\left(M_{l}\right)=\Psi_{l}$, we have

$$
\begin{aligned}
\Psi_{l}^{2} \leq & \gamma(1-\sigma)^{-\gamma} 2^{l \gamma} \Psi_{l+1} M_{l+1}^{\frac{(n+2)\left(m^{+}-m^{-}\right)}{2}-\frac{a}{2}} \rho^{-n-2} \iint_{Q_{l+1}} F(u) d x d t \\
\leq \varepsilon \Psi_{l+1}^{2}+ & \frac{1}{\varepsilon}(1-\sigma)^{-\gamma} \gamma 2^{l \gamma}(M(\theta, \tau))^{(n+2)\left(m^{+}-m^{-}\right)-a} \\
& \times \rho^{-2(n+2)}\left(\iint_{Q_{l+1}} F(u) d x d t\right)^{2}
\end{aligned}
$$

From this by iteration

$$
\begin{gathered}
\Psi^{2}(u(\bar{x}, \bar{t})) \leq \Psi_{0}^{2} \leq \varepsilon^{l} \Psi_{l}^{2}+\frac{1}{\varepsilon} \gamma(1-\sigma)^{-\gamma} \sum_{i=0}^{l-1}\left(\varepsilon 2^{\gamma}\right)^{i} \\
\times(M(\theta, \tau))^{(n+2)\left(m^{+}-m^{-}\right)-a} \rho^{-2(n+2)}\left(\iint_{Q_{l+1}} F(u) d x d t\right)^{2} .
\end{gathered}
$$


We choose $\varepsilon=2^{-\gamma-1}$ and passing to the limit as $l \rightarrow \infty$, we obtain

$$
\begin{gathered}
\left.(u(\bar{x}, \bar{t}))^{1-m^{-}+\frac{n\left(m-m^{-}\right)}{2}} F(u(\bar{x}, \bar{t}))\right) \\
\leq \gamma(1-\sigma)^{-\gamma} \rho^{-n-2}(M(\theta, \tau))^{\frac{(n+2)\left(m^{+}-m^{-}\right)}{2}} \iint_{Q_{\frac{\eta}{2}, \frac{s}{2}}(\bar{x}, \bar{t})} f(u) u^{m^{-}} d x d t .
\end{gathered}
$$

To estimate the integral on the right-hand side of (2.5) we test integral identity by $\varphi=u^{m^{-}} \zeta^{2}$, using conditions (1.4) and the Hölder inequality, we obtain

$$
\begin{aligned}
& \iiint_{Q_{\frac{\eta}{2}, \frac{s}{2}}(\bar{x}, \bar{t})} f(u) u^{m} \bar{\zeta}^{2} d x d t \leq \gamma \iint_{Q_{\frac{\eta}{2}, \frac{s}{2}}(\bar{x}, \bar{t})} u^{m^{-}+1}\left|\zeta_{t}\right| \zeta d x d t+\gamma \sum_{i=1}^{n} \underset{Q_{\frac{\eta}{2}, \frac{s}{2}}}{\int} \iint_{\bar{x}, \bar{t})} u^{m_{i}+m^{-}}\left|\zeta_{x_{i}}\right|^{2} d x d t \\
& \quad \leq \gamma \rho^{-2} M^{m^{+}+m^{-}}(\theta, \tau)\left|Q_{\frac{\eta}{2}, \frac{s}{2}}(\bar{x}, \bar{t})\right| \leq \gamma \rho^{n} M^{m^{-}+1+\frac{m-m^{+}}{2}} n(\theta, \tau) .
\end{aligned}
$$

Since $(\bar{x}, \bar{t})$ is an arbitrary point in $Q_{\sigma \theta, \sigma \tau}\left(x^{(0)}, t^{(0)}\right)$ from $(2.5),(2.6)$ we arrive at

$$
\begin{aligned}
& (M(\sigma \theta, \sigma \tau))^{1-m^{-}+\frac{n\left(m-m^{-}\right)}{2}} F(M(\sigma \theta, \sigma \tau)) \\
\leq & \gamma(1-\sigma)^{-\gamma} \rho^{-2}(M(\theta, \tau))^{m^{+}+1+\frac{n\left(m-m^{-}\right)}{2}} .
\end{aligned}
$$

For $j=0,1,2 \ldots$ define the sequences $\left\{\sigma_{j}\right\},\left\{\theta_{j}\right\},\left\{\tau_{j}\right\},\left\{M_{j}\right\}$ by $\sigma_{j}:=\frac{1-2^{-j-1}}{1-2^{-j-2}}, \theta_{j}:=\left(\theta_{1 j}, \theta_{2 j}, \ldots, \theta_{n j}\right), \theta_{i j}=\theta_{i}\left(1+\frac{1}{2}+\cdots+\frac{1}{2^{j}}\right)$, $i=\overline{1, n}, \quad \tau_{j}=\tau\left(1+\frac{1}{2}+\cdots+\frac{1}{2^{j}}\right), \quad M_{j}:=\sup _{Q_{\theta_{j}, \tau_{j}}\left(x^{(o)}\right)}$, $\Gamma\left(M_{j}\right)=\left[\frac{F\left(M_{j}\right)}{M_{j}^{m^{+}+m^{-}}}\right] \overline{\frac{1}{m^{+}+1+\frac{n\left(m-m^{-}\right)}{2}}}$.

We write (2.7) for the pair of boxes $Q_{\theta_{j}, \tau_{j}}\left(x^{(0)}, t^{(0)}\right)$ and $Q_{\theta_{j+1}, \tau_{j+1}}\left(x^{(0)}, t^{(0)}\right)$. This gives

$$
M_{j} \Gamma\left(M_{j}\right) \leq \gamma(1-\sigma)^{-\gamma} 2^{j \gamma} \rho^{\frac{-2}{m^{+}+1+\frac{n\left(m-m^{-}\right)}{2}}} M_{j+1} .
$$

Using the following inequality which is an immediate consequence of our choice of $\Gamma$

$$
\Gamma(u) v \leq \varepsilon^{-1} \Gamma(u) u+\Gamma(\varepsilon v) v, \varepsilon, u, v>0,
$$

indeed if $v \leq \varepsilon^{-1} u$, then $\Gamma(u) v \leq \varepsilon^{-1} \Gamma(u) u$, and if $v \geq \varepsilon^{-1} u$, then $\Gamma(u) v \leq \Gamma(\varepsilon v) v$, and in both cases (2.8) holds. 
If $\varepsilon \in(0,1), \mu=\frac{\beta}{m^{+}+1+\frac{n\left(m-m^{-}\right)}{2}}$ then

$$
\begin{gathered}
\Gamma\left(M_{l}\right) \leq \Gamma\left(\varepsilon M_{l+1}\right)+\frac{1}{\varepsilon} \frac{\Gamma\left(M_{l}\right) M_{l}}{M_{l+1}} \\
\leq \Gamma\left(\varepsilon M_{l+1}\right)+\varepsilon^{-1} \gamma(1-\sigma)^{-\gamma} 2^{l \gamma} \rho^{\frac{-2}{m^{+}+1+\frac{n\left(m-m^{-}\right)}{2}}} \\
\leq \varepsilon^{\mu} \Gamma\left(M_{l+1}\right)+\varepsilon^{-1} \gamma(1-\sigma)^{-\gamma} 2^{l \gamma} \rho^{\frac{-2}{m^{+}+1+\frac{n\left(m-m^{-}\right)}{2}}} .
\end{gathered}
$$

From this by iteration

$$
\Gamma\left(M_{0}\right) \leq \varepsilon^{l \mu} \Gamma\left(M_{i+1}\right)+\varepsilon^{-1} \gamma(1-\sigma)^{-\gamma} \sum_{i=0}^{l}\left(\varepsilon^{i \mu} 2^{i \gamma}\right) \rho^{\frac{-2}{m^{+}+1+\frac{n\left(m-m^{-}\right)}{2}}}
$$

We choose $\varepsilon^{\mu}=2^{-\gamma-1}$ and passing to the limit as $l \rightarrow \infty$, we obtain

$$
\Gamma\left(u\left(x^{(0)}, t^{(0)}\right)\right) \leq \gamma(1-\sigma)^{-\gamma} \rho^{\frac{-2}{m^{+}+1+\frac{n\left(m-m^{-}\right)}{2}}} .
$$

Return to the previous notation

$$
F\left(u\left(x^{(0)}, t^{(0)}\right)\right) \leq \gamma(1-\sigma)^{-\gamma}(M(\theta, \tau))^{m^{+}+m^{-}} \rho^{-2} .
$$

Thus Theorem 1.2 is proved.

\section{Proof of Theorem 1.1}

\subsection{Pointwise estimates of solutions}

Let

$$
Q_{r}=\left\{(x, t) \in \Omega_{T}:\left(t^{\frac{\kappa(\lambda)}{\kappa_{1}(\lambda)}}+\sum_{i=1}^{n}\left|x_{i}\right|^{\frac{\kappa_{i}(\lambda)}{\kappa_{1}(\lambda)}}\right)^{\kappa_{1}(\lambda)}<r,\right\}
$$

where $\kappa(\lambda)=\frac{1}{2+(n-\lambda)(m-1)}, \quad \kappa_{i}(\lambda)=\frac{2}{2+(n-\lambda)\left(m-m_{i}\right)}, i=\overline{1, n}, \lambda=n-$ $\frac{2}{q-m}$. For $0<r<\rho<\frac{R_{0}}{2}\left(R_{0}: Q_{R_{0}} \subset \Omega_{T}\right)$ we set $M(r)=\sup _{Q_{R_{0}} \backslash Q_{r}} u(x, t)$ and $u_{2 \rho}=u(x, t)-M(2 \rho) \leq M\left(\frac{\rho}{2}\right)-M(2 \rho)$ for $(x, t) \in Q_{R_{0}} \backslash Q_{\frac{\rho}{2}}$. For fixed $k>0$ and $j=0,1, \ldots$ set $\rho_{j}=\frac{\rho}{4}\left(1+\frac{1}{2^{j}}\right), k_{j}=k\left(1-2^{-j}\right)$, $A_{k_{j}, j}=\left\{(x, t) \in Q_{\rho_{j}}: u_{2 \rho}>k_{j}\right\}$. Let $\zeta_{j} \in C^{\infty}\left(Q_{\frac{\rho_{j+1}+\rho_{j}}{2}}\right), 0 \leq \zeta_{j} \leq 1$, $\zeta_{j}=1$ outside $Q_{\rho_{j}}, \zeta_{j}=0$ in $Q_{\rho_{j+1}}$, and $\left|\frac{\partial \zeta_{j}}{\partial t}\right| \leq \gamma 2^{j \gamma} \rho^{-\frac{1}{\kappa(\lambda)}},\left|\frac{\partial \zeta_{j}}{\partial x_{i}}\right| \leq$ 
$\gamma 2^{j \gamma} \rho^{-\frac{2}{\kappa_{i}(\lambda)}}, i=\overline{1, n}$. Let $i_{0}$ be the number such that $m_{i} \leq 1, i=1, \ldots i_{0}$ and $m_{i}>1, i=i_{0}+1, \ldots n, m^{\prime}=\frac{1}{n} \sum_{i=1}^{i_{0}} m_{i}, m^{\prime \prime}=\frac{1}{n} \sum_{i=i_{0}+1}^{n} m_{i}$. Note that $i_{0}=0$ if $m_{i}>1, i=\overline{1, n}$, and $i_{0}=n$ if $m_{i} \leq 1, i=\overline{1, n}$.

Testing identity (1.4) by $\varphi=\left(u_{2 \rho}-k_{j}\right)_{+} \zeta_{j}^{2}$, using conditions (1.4) we obtain

$$
\begin{aligned}
& \operatorname{ess} \sup \int_{A_{k_{j}, j}(t)}\left(u_{2 \rho}-k_{j}\right)_{+}^{2} \zeta_{j} d x+\sum_{i=1}^{i_{0}} M^{m_{i}-1}\left(\frac{\rho}{2}\right) \iint_{A_{k_{j}, j}}\left|u_{x_{i}}\right|^{2} \zeta_{j}^{2} d x d t \\
& \quad+\sum_{i=i_{0}+1}^{n} k_{j}^{m_{i}-1} \iint_{A_{k_{j}, j}}\left|u_{x_{i}}\right|^{2} \zeta_{j}^{2} d x d t+\iint_{A_{k_{j}, j}}\left(u_{2 \rho}-k_{j}\right)_{+} u^{q} \zeta_{j}^{2} d x d t \\
& \leq \gamma\left(M^{2}\left(\frac{\rho}{2}\right) \rho^{-\frac{1}{\kappa(\lambda)}}+\sum_{i=1}^{n} M^{m_{i}+1}\left(\frac{\rho}{2}\right) \rho^{-\frac{2}{\kappa_{i}(\lambda)}}\right)\left|A_{k_{j}, j}\right| .
\end{aligned}
$$

By Lemma 2.2, the Hölder inequality and estimate (3.1), we obtain

$$
\begin{gathered}
Y_{j+1}=\iint_{A_{k_{j+1}, j+1}}\left(u_{2 \rho}-k_{j+1}\right)_{+}^{2} d x d t \\
\leq\left|A_{k_{j+1}, j+1}\right|^{\frac{2}{n+2}}\left(\int_{A_{k_{j+1}, j+1}}\left(\left(u_{2 \rho}-k_{j+1}\right)_{+} \zeta_{j}\right)^{2+\frac{4}{n}} d x d t\right)^{\frac{n}{n+2}} \\
\leq\left|A_{k_{j+1}, j+1}\right|_{0<t<T}^{\frac{2}{n+2}} \operatorname{ess~sup}_{0}\left(\int_{A_{k_{j+1}, j+1}(t)}\left(u_{2 \rho}-k_{j+1}\right)_{+}^{2} \zeta_{j}^{2} d x\right)^{\frac{2}{n+2}} \\
\times\left(\int_{0}^{t} \prod_{i=1}^{n}\left(\int_{A_{k_{j+1}, j+1}(t)}\left|\left(\left(u_{2 \rho}-k_{j+1}\right)_{+} \zeta_{j}\right)_{x_{i}}\right|^{2} d x\right)^{\frac{1}{n}} d \tau\right)^{\frac{n}{n+2}} \\
\times\left(M^{2}\left(\frac{\rho}{2}\right) \rho^{-\frac{1}{\kappa(\lambda)}}+\sum_{i=1}^{n} M^{m_{i}+1}\left(\frac{\rho}{2}\right) \rho^{-\frac{2}{\kappa_{i}(\lambda)}}\right)\left|A_{k_{j+1}, j+1}\right|^{1+\frac{2}{n+2}}
\end{gathered}
$$

From this by the evident inequality $\left(u_{2 \rho}-k_{j}\right)_{+} \geq \frac{k}{2^{j+1}}$ on $A_{k_{j+1}, j}$, we obtain estimate 


$$
\begin{gathered}
Y_{j+1} \leq \gamma 2^{j \gamma} M^{\frac{\left(1-m^{\prime}\right) i_{0}}{n+2}}\left(\frac{\rho}{2}\right) k_{j+1}^{\frac{\left(1-m^{\prime \prime}\right)\left(n-i_{0}\right)}{n+2}}\left(M^{2}\left(\frac{\rho}{2}\right) \rho^{-\frac{1}{\kappa(\lambda)}}\right. \\
\left.+\sum_{i=1}^{n} M^{m_{i}+1}\left(\frac{\rho}{2}\right) \rho^{-\frac{2}{\kappa_{i}(\lambda)}}\right) Y_{j}^{1+\frac{2}{n+2}}
\end{gathered}
$$

It follows from Lemma 2.3 that

$$
\begin{gathered}
(M(\rho)-M(2 \rho))^{\frac{\left(m^{\prime \prime}-1\right)\left(n-i_{0}\right)}{2}+n+4} \leq \gamma 2^{j \gamma} M^{\frac{\left(1-m^{\prime}\right) i_{0}}{n+2}}\left(\frac{\rho}{2}\right) \\
\times\left(M^{2}\left(\frac{\rho}{2}\right) \rho^{-\frac{1}{\kappa(\lambda)}}+\sum_{i=1}^{n} M^{m_{i}+1}\left(\frac{\rho}{2}\right) \rho^{-\frac{2}{\kappa_{i}(\lambda)}}\right) \iint_{Q \frac{\rho}{2}} u_{2 \rho}^{2} d x d t .
\end{gathered}
$$

By the Hölder inequality and Lemma 2.1 we get

$$
\begin{gathered}
(M(\rho)-M(2 \rho))^{\frac{\left(m^{\prime \prime}-1\right)\left(n-i_{0}\right)}{2}+n+4} \leq \gamma 2^{j \gamma} M^{\frac{\left(1-m^{\prime}\right) i_{0}}{n+2}}\left(\frac{\rho}{2}\right) \\
\times\left(M^{2}\left(\frac{\rho}{2}\right) \rho^{-\frac{1}{\kappa(\lambda)}}+\sum_{i=1}^{n} M^{m_{i}+1}\left(\frac{\rho}{2}\right) \rho^{-\frac{2}{\kappa_{i}(\lambda)}}\right) \\
\times\left\{F_{3}(r, \lambda)+\left(F_{1}(r, \lambda)+F_{2}(r, \lambda)\right)^{\frac{1}{2}} F_{4}^{\frac{1}{2}}(r, \lambda)\right\}\left|Q_{\frac{\rho}{2}}\right|^{\frac{q-1}{q+1}} .
\end{gathered}
$$

Similarly to [11], we obtain the following estimate

$$
M(\rho)-M(2 \rho) \leq 0,
$$

iterating last inequality we get for any $\rho \leq \frac{R_{0}}{2}$

$$
M(\rho) \leq M\left(R_{0}\right),
$$

this proves the boundedness of solutions.

\subsection{End of the proof of Theorem 1.1}

Let $K$ be a compact subset in $\Omega$, and $\xi=0$ in $\partial \Omega \times(0, T)$, such that $\xi=1$ for $(x, t) \in K \times(0, T)$. Testing (1.5) by $\varphi=u^{m^{-}} \xi^{2} \psi_{r}, \psi=\psi_{r}$, using conditions (1.3), the Young inequality, the boundedness of $u$ and passing to the limit $r \rightarrow 0$ we get

$$
\sup _{0<t<T} \int_{K} u^{m^{-}+1} d x+\sum_{i=1}^{n} \int_{0}^{T} \int_{K} u^{m_{i}+m^{-}-2}\left|u_{x_{i}}\right|^{2} d x d t+\int_{0}^{T} \int_{K} u^{q+m^{-}} d x d t \leq \gamma .
$$


Testing (1.5) by $\varphi \psi_{r}$, using (1.3), the boundedness of solution, and passing to the limit $r \rightarrow 0$, we obtain the integral identity (1.5) with an arbitrary $\varphi \in W_{l o c}^{1,2}\left(0, T ; L_{l o c}^{2}(\Omega)\right) \cap L_{l o c}^{2}\left(0, T ; \stackrel{o}{W_{l o c}^{1,2}}(\Omega)\right)$ and $\psi \equiv 1$. Thus Theorem 1.1 is proved.

\section{Acknowledgements}

This paper is supported by Ministry of Education and Science of Ukraine, grant number is $0118 \mathrm{U} 003138$.

\section{References}

[1] O. V. Besov, V. P. Ilin, S. M. Nikolskii, Integral representations of functions and embedding theorems, New York Toronto, 1978.

[2] H. Brezis, L. Veron, Removable singularities for some nonlinear elliptic equations // Arch. Rational Mech. Anal., 75(1) (1980), 1-6.

[3] H. Brezis, A. Friedman, Nonlinear parabolic equations involving measure as initial conditions // J. Math. Pures Appl., 62 (1983), 73-97.

[4] N. Fusco, C. Sbordone, Some remarks on the regularity of minima of anisotropic integrals // Comm. PDE, 18 (1993), 153-167.

[5] S. Kamin, L. A. Peletier, Source type solutions of degenerate diffusion equations with absorption // Israel JI. Math., 50 (1985), 219-230.

[6] I. M. Kolodij, On boundedness of generalized solutions of parabolic differential equations // Vestnik Moskov. Gos. Univ., 5 (1971), 25-31.

[7] G. Lieberman, Gradient estimates for anisotropic elliptic equations // Adv. Diff. Equat., 10 (2005), No. 7, 767-812.

[8] O. A. Ladyzhenskaya, N. N. Ural'tseva, Linear and quasilinear elliptic equations, Academic Press, New York, 1968.

[9] Yu. V. Namlyeyeva, A. E. Shishkov, I. I. Skrypnik, Removable isolated singularities for solutions of doubly nonlinear anisotropic parabolic equations // Appl. Analysis, 89(10) (2010), No. 4, 1559-1574.

[10] Yu. V. Namlyeyeva, A. E. Shishkov, I. I. Skrypnik, Isolated singularities of solutions of quasilinear anisotropic elliptic equations // Adv. Nonlin. Studies, 6 (2006), No. 4, 617-641.

[11] M. A. Shan, Removability of an isolated singularity for solutions of anisotropic porous medium equation with absorption term // J. Math. Sciences, 222 (2017), No. 6, 741-749.

[12] M. A. Shan, I. I. Skrypnik, Keller-Osserman a priori estimates and the Harnack inequality for quasilinear elliptic and parabolic equations with absorption term // Nonlinear Analysis, 155 (2017), 97-114.

[13] I. I. Skrypnik, Local behaviour of solutions of quasilinear elliptic equations with absorption // Trudy Inst. Mat. Mekh. Nats. Akad. Nauk Ukrainy, 9 (2004), 183190 [in Russian].

[14] I. I. Skrypnik, Removability of isolated singularities of solutions of quasilinear parabolic equations with absorption // Mat. Sb., 196 (2005), No. 11, 141-160; transl. in Sb. Math., 196 (2005), No. 11, 1693-1713. 
[15] I. I. Skrypnik, Removability of an isolated singularity for anisotropic elliptic equations with absorption // Mat. Sb., 199 (2008), No. 7, 8-102.

[16] I. I. Skrypnik, Removability of isolated singularity for anisotropic parabolic equations with absorption // Manuscr. Math., 140 (2013), 145-178.

[17] I. I. Skrypnik, Removability of isolated singularities for anisotropic elliptic equations with gradient absorption // Isr. J. Math., 215 (2016), 163-179.

[18] I. I. Skrypnik, Removable singularities for anisotropic elliptic equations // Isr. J. Math., 41 (2014), No. 4, 1127-1145.

[19] L. Veron, Singularities of solution of second order quasilinear equations, Pitman Research Notes in Mathematics Series, Longman, Harlow, 1996.

\section{CONTACT INFORMATION}

Maria Alekseevna Vasyl' Stus Donetsk National University,

Shan Vinnytsia, Ukraine E-Mail: shan_maria@ukr.net 